\title{
Silicon dual-ring modulator driven by differential signal
}

\author{
Hui Yu, ${ }^{1,3, *}$ Marianna Pantouvaki, ${ }^{2}$ Peter Verheyen, ${ }^{2}$ Guy Lepage, ${ }^{2}$ \\ Philippe Absil, ${ }^{2}$ Wim Bogaerts, ${ }^{2}$ and Joris Van Campenhout ${ }^{2}$ \\ ${ }^{1}$ Photonics Research Group, Department of Information Technology, Ghent University-imec, Center for Nano- and Biophotonics \\ (NB photonics), St.-Pietersnieuwstraat 41, Gent 9000, Belgium \\ ${ }^{2}$ imec, Kapeldreef 75, Leuven 3001, Belgium \\ ${ }^{3}$ Department of Information Science and Electronic Engineering, Zhejiang University, Hangzhou 310027, China \\ *Corresponding author: huiyu@zju.edu.cn
}

Received August 11, 2014; accepted October 1, 2014; posted October 14, 2014 (Doc. ID 220826); published November 4, 2014

A silicon dual-ring modulator consisting of two serially cascaded rings with embedded PN junctions is driven by a differential signal pair. We show by simulation and experiment that the device has advantages over the single-ring modulator in terms of optical bandwidth, 3-dB modulation bandwidth and bit rate, at the expense of a 1.7-dB increase in the transmission penalty and a twofold increase of the RF power consumption. Driven by differential pseudo random binary sequence (PRBS) signals of $0.5-\mathrm{V}$ peak-to-peak voltage $\left(\mathrm{V}_{\mathrm{pp}}\right)$, the dual-ring modulator exhibits optical bandwidths of $66 \mathrm{pm}$ and $40 \mathrm{pm}$ at $12.5 \mathrm{~Gb} / \mathrm{s}$ and $20 \mathrm{~Gb} / \mathrm{s}$, respectively. In contrast, the single-ring modulator has an optical bandwidth of $26 \mathrm{pm}$ under a single-end PRBS signal of $0.5 \mathrm{~V}_{\mathrm{pp}}$ at $12.5 \mathrm{~Gb} / \mathrm{s}$, and its eye diagram closes if the bit rate rises to $20 \mathrm{~Gb} / \mathrm{s}$. (c) 2014 Optical Society of America

OCIS codes: (230.4110) Modulators; (230.4555) Coupled resonators; (230.3120) Integrated optics devices.

http://dx.doi.org/10.1364/OL.39.006379

Silicon carrier-depletion-based ring modulators are able to meet stringent requirements of optical interconnect systems in terms of footprint, driving voltage, power consumption, CMOS compatibility, and so on. It is well-known that the bandwidth of a ring modulator is subjected to two limitations: the RC constant of the $\mathrm{PN}$ junction and the resonance linewidth of the optical cavity. The latter becomes the major limitation to the bandwidths of many successfully demonstrated highperformance devices [1-3]. A straightforward way to mitigate this constraint is to reduce the quality factor $\mathrm{Q}$ of the ring. However, this is adverse to the electro-optical (EO) modulation since a low-Q ring is less sensitive to any variation of the refractive index. To solve this issue, the forward-biased PIN diode, which has a much higher modulation efficiency than the reverse biased PN diode, is used to drive a low-Q ring in [4]. Another approach to enhance the modulation depth of a low-Q resonator is to cascade two rings in parallel [5]. This configuration enables a steeper resonance lineshape than the single ring of the same resonance linewidth. Therefore, a higher extinction ratio (ER) can be obtained with the same refractive index shift. Besides the intracavity modulation, which changes the phase of the circling light, the modulation of the coupling strength between the ring and the bus waveguide has been also investigated to overcome the bandwidth limitation imposed by the cavity linewidth. A modulation bandwidth of $6 \times$ the cavity linewidth is obtained in [6].

By serially coupling two ring resonators, various functionalities have been demonstrated, including broadband hitless switch [7], reconfigurable switch [8], vernier resonator [9], and so on. In [10], Li proposes a modulator based on this structure. In his scheme, only the inner ring immediately next to the bus waveguide is actively driven. Theoretical analysis indicates that the modulation speed is faster than that of the single-ring modulator. On the other hand, Zortman uses a differential signaling pair to drive a microdisk modulator and get a power consumption of $3 \mathrm{fJ} / \mathrm{bit}$ at $10 \mathrm{~Gb} / \mathrm{s}$ [11]. Although it does not improve metrics such as ER, speed, or power consumption, the differential signaling is inherently compatible with the low-voltage signaling technique (LVS), which is likely to be implemented on future chip designs. In this Letter, we combine the serially cascaded dual-ring modulator with the differential signaling, i.e., two complementary signals of a differential pair feed to the two coupled rings separately. A comprehensive comparison with the single-ring modulator on the same chip shows that when driven by a differential signal pair, the dual-ring modulator has many advantages over the single-ring modulator.

A schematic diagram of the dual-ring modulator and its microscope image are presented in Figs. 1(a) and 1(b), respectively. Each ring contains a PN junction embedded inside a rib waveguide and a tungsten heater on top of that, as shown in Fig. 1(c). In order to share the same patterning steps as the fiber grating coupler [12], the etching depth of the 450-nm-wide rib waveguide is $70 \mathrm{~nm}$, which constrains the ring radius to be $40 \mu \mathrm{m}$. A sharper bend can be implemented if we enhance the lateral confinement by increasing the etching depth, however, at the cost of additional litho and etching steps.

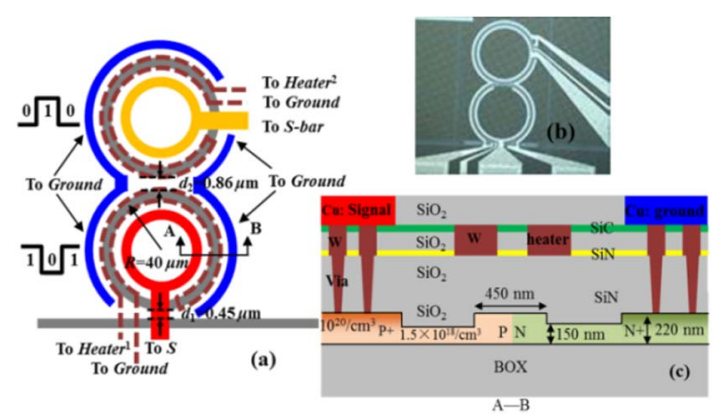

Fig. 1. Schematic diagram (a) and microscope image (b) of the dual-ring modulator. (c) Cross section of the ring waveguide, which contains a PN junction and a tungsten heater. 
The nominal doping concentration of the $\mathrm{PN}$ junctions is $1.5 \times 10^{18} / \mathrm{cm}^{3}$. The ohmic contact regions besides the rib waveguide are heavily doped to $1 \times 10^{20} / \mathrm{cm}^{3}$. Two complementary driving signals, i.e., $S$ and $S$-bar, are applied on the PN junctions of the bottom and the top rings, respectively. Two tungsten heaters based on standard CMOS back-end fabrication processing as introduced in [13] can tune the two rings independently. The gap between the bottom ring and the bus waveguide is $0.45 \mu \mathrm{m}$, while that between the two rings is $0.86 \mu \mathrm{m}$. A reference single-ring modulator is situated next to the dual-ring modulator. It has the same design parameters as the dual-ring modulator except that the gap between the ring and the bus waveguide is $0.5 \mu \mathrm{m}$ for critical coupling. The performance of this reference device has been discussed in our previous work [14]. However, some relevant data will be presented again so as to compare with the dualring modulator. Device fabrication is carried out by imec's silicon photonics platform (iSipp) based on its 200-mm CMOS pilot line. Detailed descriptions regarding the processing flow can be found in $[13,15]$.

Measured spectra at the through port of the single-ring modulator under different bias voltages are depicted as discrete points in Fig. 2(a). The resonance wavelength, the cross-coupling coefficient $K$ between the ring and the bus waveguide, and the beam propagation loss in the ring can be extracted by curve fitting to these data. The fitted resonance curves are presented in Fig. 2(a) as solid lines. The value of $K$ is 0.303 for the $0.5-\mu \mathrm{m}$-wide coupling gap. As shown in Fig. 2(a), the single-ring modulator is very close to critical coupling. Its quality factor is 12,900 at zero bias. With the bias varying from $0.5 \mathrm{~V}$ to
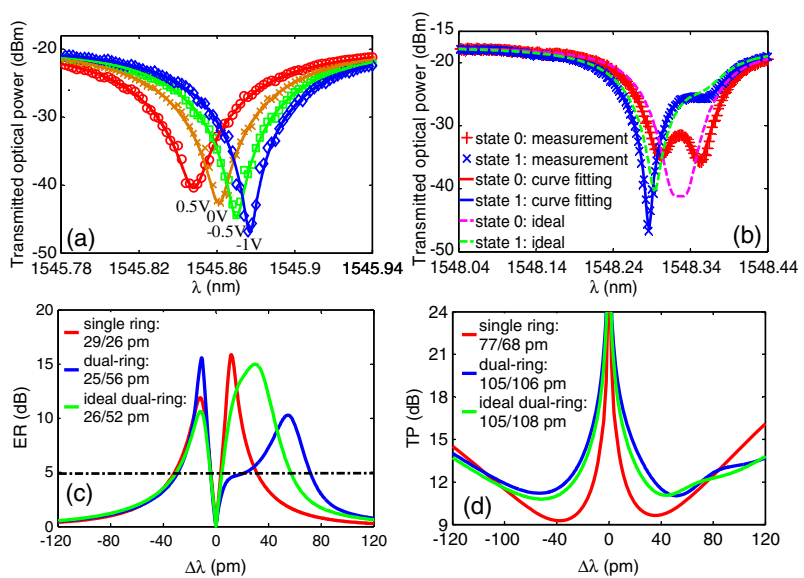

Fig. 2. (a) Measured transmission spectra of the single-ring modulator with different bias voltages. (b) Measured transmission spectra of the dual-ring modulator for $S=-S$-bar $=$ $\pm 0.5 \mathrm{~V}$ and $6.75 \mathrm{~mW}$ heater power. Dashed lines depict the calculated spectra of the dual-ring modulator if the coupling coefficient $K_{2}$ between its two rings is optimized. (c) ER of the single ring, the practical dual-ring, and the ideal dual-ring modulators versus the wavelength. Values in the legend indicate the wavelength spans of the left/right operation bands where $\mathrm{ER}>5 \mathrm{~dB}$. (d) TP of the single ring, the practical dual-ring, and the ideal dual-ring modulators versus the wavelength. Values in the legend indicate the wavelength spans of the left/right operation bands where TP rises by $3 \mathrm{~dB}$ from the bottom. In (c) and (d), both the single-end and the differential signals swing between $-0.5 \mathrm{~V}$ and $0.5 \mathrm{~V}$.
$-0.5 \mathrm{~V}$, the resonance wavelength shifts by $22 \mathrm{pm}$, which is equal to a figure of merit of $V_{\pi} L_{\pi}=1.45 \mathrm{~V} \cdot \mathrm{cm}$. Metrics to evaluate the performance of an intensity modulator include ER, which refers to the ratio of the optical power transmitted at "ON" state to that at "OFF" state (ER). Besides ER, another useful metric is the transmission penalty (TP) [16]. Its definition is $\mathrm{TP}=-10 \log _{10}\left[\left(P_{1}-\right.\right.$ $\left.\left.P_{0}\right) / 2 / P_{\text {in }}\right]$, where $P_{1}, P_{0}$ and $P_{\text {in }}$ denote the optical power transmitted at logical high, logical low, and the input power, respectively. TP denotes the relative optical modulation amplitude with respect to the input power. $\mathrm{ER}$ and TP of the single-ring modulator for a voltage swing from $-0.5 \mathrm{~V}$ to $0.5 \mathrm{~V}$ are plotted in Figs. 2(c) and $2(\mathrm{~d})$, where $\Delta \lambda$ is the wavelength offset from the point of zero modulation, i.e., the point where the spectrum of $-0.5 \mathrm{~V}$ bias intersects with that of $0.5 \mathrm{~V}$ bias.

The operation principle of the dual-ring modulator is to mismatch the resonance wavelengths of the two rings, which are initially well aligned by the differential signal pair. When both $S$ and $S$-bar swing between $-0.5 \mathrm{~V}$ and $0.5 \mathrm{~V}$, at least one heater is adjusted so that the two rings have the same resonance wavelength at either state " 0 " or state " 1 ." Here, states " 0 " and " 1 " refer to $S=-S$-bar $=$ $-0.5 \mathrm{~V}$ and $S=-S$-bar $=0.5 \mathrm{~V}$, respectively. As the differential signal switches to the other state, the two rings experience a refractive index difference, which is two times as high as that induced by a voltage swing of $1 \mathrm{~V}$.

The transmission spectra of the dual-ring modulator under states " 0 " and " 1 " are depicted in Fig. 2(b) as discrete points. To balance the opposite biases on the two rings and align their resonance wavelengths at state " 0 ," a voltage of $1.73 \mathrm{~V}$ is applied on the heater of the top ring [8]. The resulting current through the heater is $3.9 \mathrm{~mA}$, which corresponds to a heater power of $6.75 \mathrm{~mW}$. The symmetrical spectrum at state " 0 " implies that the two rings induce the same round-trip phase change to the light propagating inside [17]. We extract the crosscoupling coefficients $K_{1}$ between the ring and the bus waveguide, and $K_{2}$ between the two rings from the spectra in Fig. 2(b) by curve fitting. Their values are $K_{1}=$ 0.4579 and $\overline{K_{2}}=0.0905$. During the curving fitting, the modulation efficiency of the single ring is used, since the dual- and the single-ring modulators have the same $\mathrm{PN}$ junction design. However, the beam propagation loss in the dual-ring modulator needs to be 1.5 times as high as that in the single ring to get a good fitting. This is due to two factors: the dual-ring structure has two coupling sections, and its coupling gap between the bottom ring and the bus waveguide is narrower than that of the single-ring modulator. Both factors increase the coupling loss and the resultant round trip loss. The fitted curves are shown as solid lines in Fig. 2(b), and they have very good overlap with the measurement. We note that the spectrum at state " 0 " splits into two notches at its bottom. This is because the coupling strength between the two rings is larger than the designed value [18]. Dashed lines in Fig. 2(b) depict the spectra of the ideal dual-ring modulator at the two states if the value of $K_{2}$ falls from 0.0905 to 0.0663 .

In Figs. 2(c) and 2(d), ER and TP of the ideal dual-ring modulator are plotted for a differential signal pair of $1 \mathrm{~V}$. It is shown that the ideal dual-ring modulator has a larger optical bandwidth than the single-ring modulator. Since 
there is no explicit definition of the optical bandwidth of ring modulators, we compare both the wavelength band where the ER exceeds $5 \mathrm{~dB}$, and the wavelength band where TP rises by $3 \mathrm{~dB}$ from the bottom. Both singleand dual-ring modulators have two operation bands. The spans of the left/right bands where $\mathrm{ER}>5 \mathrm{~dB}$ are $29 / 26 \mathrm{pm}$ for the single ring, and 26/52 pm for the ideal dual-ring. Therefore, the right band of the ideal dual-ring modulator is around twice the width of either bands of the single-ring counterpart. Moreover, the 3-dB bandwidths of TP at the left/right bands are 105/108 pm and $77 / 68 \mathrm{pm}$ for the ideal dual ring and the single ring, respectively.

As aforementioned, $K_{2}$ of the practice device is 0.0905 instead of the ideal value of 0.0663 due to the fabrication imperfection. As a consequence, the maximum ER on the right band falls by $\sim 5 \mathrm{~dB}$ as shown in Fig. 2(c). However, this nonideal coupling coefficient has little impact on the bandwidth and on TP. For the left/right operation bands of the practical device, the wavelength spans of $\mathrm{ER}>5 \mathrm{~dB}$ are $25 / 56 \mathrm{pm}$, whilst the corresponding $3-\mathrm{dB}$ bandwidths of TP are 105/106 pm. These values are very close to the ideal case.

The comparison above confirms that the dual-ring modulator driven by a differential signal pair indeed possesses a wider optical bandwidth. However, as can be seen in Fig. 2(d), this improvement comes with a penalty of $\sim 1.7 \mathrm{~dB}$ in the minimum TP. A wider bandwidth implies that the modulation is less susceptible to any fluctuation of the environmental temperature, and therefore relaxes the design requirement for the thermal stabilization circuit. More importantly, the optical bandwidth, i.e., the cavity linewidth, is associated with the EO modulation bandwidth through the ring dynamics [14]. A wider optical bandwidth leads to a higher modulation speed, as will be discussed in the following.

The single-ring modulator in Fig. 2(a) used to compare with the dual-ring modulator is close to critical coupling. We note that increasing its cross-coupling coefficient $K$ could reduce the quality factor, and consequently broaden the 3-dB bandwidth of TP. However, this approach to improve the bandwidth of TP is adverse to the ER since the ring is over-coupled. For example, if the value of $K$ rises from 0.303 in Fig. 2(a) to 0.394, the resultant 3-dB bandwidths of TP at the left/right operation bands increase to $107 / 74 \mathrm{pm}$, which are comparable with those of the dual-ring modulator. Meanwhile the maximum ER at the left/right bands fall to $5.6 / 4 \mathrm{~dB}$, which are inferior to those of the dual-ring modulator.

The operation speed of ring modulators is limited by the $\mathrm{RC}$ constant of the PN junction as well as the cavity linewidth. Since both rings in the dual-ring modulator are identical to the single-ring modulator, electrically these diode-embedded rings can be represented by the same lumped circuit as shown in Fig. 3(b), where $C_{0}, C_{1}$, $C_{2}, R_{1}$, and $R_{2}$ represent the pad capacitance, the PN junction capacitance, the buried oxide induced capacitance, and the series resistances of the PN junction and of the substrate, respectively. Figure 3(a) shows the S11 measurement and fitting versus frequency. The values of $C_{0}, C_{1}, C_{2}, R_{1}$, and $R_{2}$ resulting from the fitting to the measured data are $20 \mathrm{fF}, 170 \mathrm{fF}, 400 \mathrm{fF}, 75 \Omega$, and

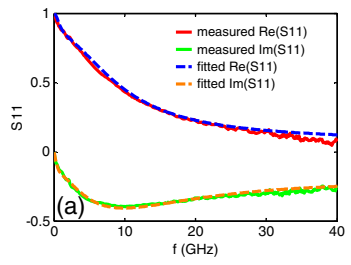

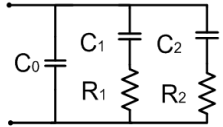

(b)
Fig. 3. (a) S11 parameter of the single-ring modulator with $0 \mathrm{~V}$ bias. (b) The equivalent circuit used to describe the single-ring modulator.

$1500 \Omega$, respectively. Taking the $50-\Omega$ internal impedance of the measurement system into account, the $\mathrm{AC}$ voltage on $C_{1}$, which is responsible for EO modulation, has a $3-\mathrm{dB}$ bandwidth of $f_{\mathrm{RC}}=7 \mathrm{GHz}$. This limitation is equal for both single and dual ring.

We have established an analytic model that can calculate the small-signal frequency response of the single-ring modulator from its equivalent circuit and the static transmission spectra [14]. Here we extend this model so that it is applicable to the dual-ring modulator. Based on the spectra of Fig. 2 and the circuit of Fig. 3(b), we use the model to calculate $|S 21|^{2}$ of the single-ring modulator, the dual-ring modulator driven by a single-end signal on its bottom ring (the scheme proposed in [10]), and by a differential signal pair on both rings. The results are presented as dashed lines in Fig. 4(a). Our experimental setup only allows us to feed a single-end sinusoid RF signal to the devices. Therefore, only $|S 21|^{2}$ of the single-ring and the dual-ring modulators with the single-end signal are measured. Black solid lines in Fig. 4(a) present the measurement result. The good agreement between the measurement and the theoretical calculation proves the validity of the model. In Fig. 4(b), we model the frequency response of the dual-ring modulator as its $\mathrm{PN}$ junction capacitance $C_{1}$ decreases from 170 to $70 \mathrm{fF}$ while other parameters remain the same as in Fig. 4(a).

In Fig. 4(b), the strength of $|S 21|^{2}$ is enhanced significantly at high frequency by using a differential signal to drive the dual ring. As a result, the $3-\mathrm{dB}$ modulation bandwidth of this scheme is $13 \mathrm{GHz}$ higher than that of the single ring, i.e., from $9.2 \mathrm{GHz}$ for the single ring
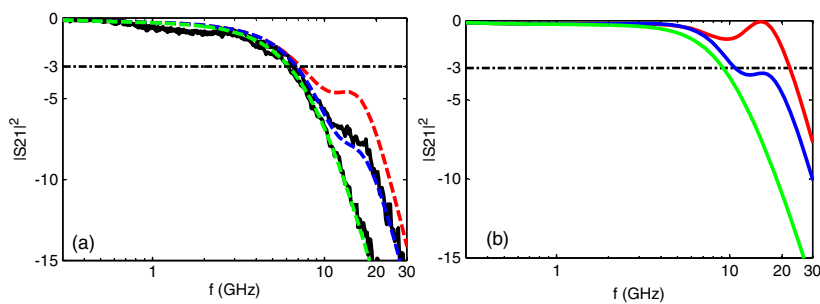

Fig. 4. (a) Measurement and modeling result of $|S 21|^{2}$ of the single- and the dual-ring modulators. The green, blue, and red dashed curves are modeled $|S 21|^{2}$ of the single-ring modulator, the dual-ring modulator driven by a single-end signal, and driven by a differential signal pair, respectively. The two black solid curves display the corresponding measurement result. (b) modeling result of $|S 21|^{2}$ with a reduced PN junction capacitance of $C_{1}=70 \mathrm{fF}$. The green, blue, and red solid curves correspond to the single-ring modulator, the dual-ring modulator driven by a single-end signal, and driven by a differential signal pair, respectively. 


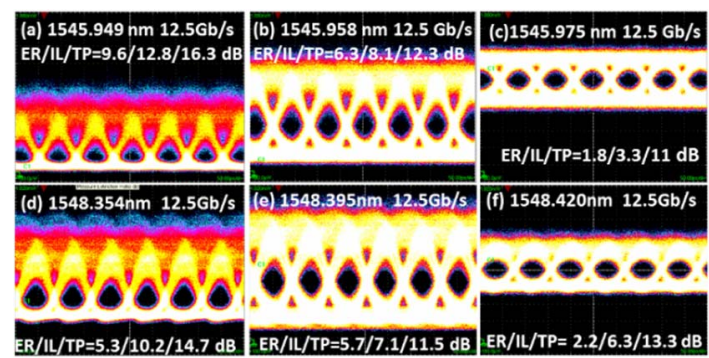

Fig. 5. Eye diagrams of the single-ring (a)-(c) and the dualring (d)-(f) modulators at $12.5 \mathrm{~Gb} / \mathrm{s}$. The pattern length and the peak-to-peak voltage of both the single-end and the differential PRBS signals are 2E31- 1 and $0.5 \mathrm{~V}_{\mathrm{pp}}$, respectively. DC biases added on the single-end and the differential signals are -0.2 and $0 \mathrm{~V}$, respectively.

to $22.2 \mathrm{GHz}$ for the dual ring. However, a large $\mathrm{RC}$ time constant can suppress this advantage to some extent. The reason is that the $\mathrm{RC}$ time constant becomes the major limitation to the modulation speed as it increases. Although the dual ring relaxes the bandwidth limitation resulting from the cavity linewidth, it does not affect the circuit in Fig. 3(b). This point is illustrated in Fig. 4(a), where the practical dual ring of $C_{1}=170 \mathrm{fF}$ under a single-end signal exhibits a $3-\mathrm{dB}$ bandwidth of $7.2 \mathrm{GHz}$, only $1 \mathrm{GHz}$ higher than that of the practical single ring $(6.2 \mathrm{GHz})$.

After the S21 measurement, we compare the eye diagrams of the single- and the dual-ring modulators. The evolution of the eye diagram versus wavelength is shown in Fig. 5 for both modulators at a bit rate of $12.5 \mathrm{~Gb} / \mathrm{s}$. The input single-end PRBS signal on the single ring has a peak-to-peak voltage of $0.5 \mathrm{~V}$. Due to the DC bias of $-0.2 \mathrm{~V}$ and the $\mathrm{RF}$ reflection, the voltage on the diode swings between -0.7 and $0.3 \mathrm{~V}$. The single-ring modulator provides clear open eyes within a range of $26 \mathrm{pm}$. In contrast, the dual-ring modulator, which is driven by a differential PRBS signal pair of $0.5 \mathrm{~V}_{\mathrm{pp}}$ without any DC bias, supports open eyes within a span of $66 \mathrm{pm}$. This value is more than twice that of the single-ring modulator. In addition, the dual-ring modulator operates well under the same driving condition at $20 \mathrm{~Gb} / \mathrm{s}$ within a wavelength span of $40 \mathrm{pm}$, whereas the eye of the single-ring modulator is almost closed at this bit rate as shown in Fig. $\underline{6}$. The dynamic IL in Figs. $\underline{5}$ and $\underline{6}$ corresponds to the transmitted optical power at logical high. It can be seen that at their favorable wavelengths, the single ring in Fig. 5(b) and the dual ring in Fig. 5(e) exhibit comparable IL. Comparison of the eye diagrams confirms that the dual-ring modulator indeed has higher optical bandwidth and operation speed. However, compared with the single ring, the RF power consumption of the dual-ring modulator doubles since both rings of it are actively driven.

In conclusion, in this Letter, we show that the optical bandwidth and the modulation speed of the ring-based modulator can be improved by using a differential signal on a serially cascaded dual ring, at the expense of 1.7-dB increase in TP. An improvement in the 3 - $\mathrm{dB}$ modulation bandwidth of $13 \mathrm{GHz}$ is theoretically predicted for dualring modulators with low $\mathrm{RC}$ time constant. The dual-ring

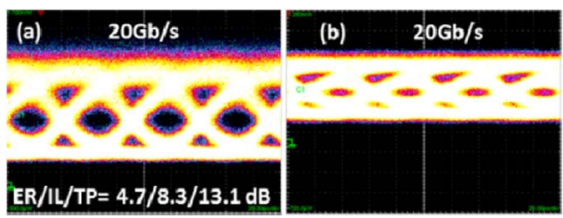

Fig. 6. Eye diagrams of (a) the dual- and (b) the single-ring modulators at $20 \mathrm{~Gb} / \mathrm{s}$. Other driving conditions are the same as those in Fig. 5 .

scheme presented here has therefore the potential to relax the need for tight wavelength control and to improve the speed of silicon ring modulators.

\section{References}

1. X. Xiao, X. Li, H. Xu, Y. Hu, K. Xiong, Z. Li, T. Chu, J. Yu, and Y. Yu, IEEE Photon. Technol. Lett. 24, 1712 (2012).

2. A. Biberman, E. Timurdogan, W. Zortman, D. C. Trotter, and M. R. Watts, Opt. Express 20, 29223 (2012).

3. G. Li, X. Zheng, H. Thacker, J. Yao, Y. Luo, I. Shubin, K. Raj, J. E. Cunningham, and A. V. Krishnamoorthy, in Proceedings of 9th IEEE International Conference on Group IV Photonics (IEEE 2012), pp. 1-3.

4. T. Baba, S. Akiyama, M. Imai, N. Hirayama, H. Takahashi, Y. Noguchi, T. Horikawa, and T. Usuki, Opt. Express 21, 11869 (2013).

5. Y. Hu, X. Xiao, H. Xu, X. Li, K. Xiong, Z. Li, T. Chu, Y. Yu, and J. Yu, Opt. Express 20, 15079 (2012).

6. W. D. Sacher, W. M. J. Green, S. Assefa, T. Barwicz, H. Pan, S. M. Shank, Y. A. Vlasov, and J. K. S. Poon, Opt. Express 21, 9722 (2013).

7. H. L. R. Lira, S. Manipatruni, and M. Lipson, Opt. Express 17, 22271 (2009).

8. W. S. Fegadolli, G. Vargas, X. Wang, F. Valini, L. A. M. Barea, J. E. B. Oliveira, N. Frateschi, A. Scherer, V. R. Almeida, and R. R. Panepucci, Opt. Express 20, 14722 (2012).

9. R. Boeck, J. Flueckiger, H. Yun, L. Chrostowski, and N. A. F. Jaeger, Opt. Lett. 37, 5199 (2012).

10. Y. Li, L. Zhang, M. Song, B. Zhang, J. Y. Yang, R. G. Beausoleil, A. E. Willner, and P. D. Dapkus, Opt. Express 16, 13342 (2008).

11. W. A. Zortman, A. L. Lentine, D. C. Trotter, and M. R. Watts, Opt. Express 19, 26017 (2011).

12. G. Roelkens and D. Vermeulen, IEEE J. Sel. Top. Quantum Electron. 17, 571 (2011).

13. A. Masood, M. Pantouvaki, D. Goossens, G. Lepage, P. Verheyen, D. Van Thourhout, P. Absil, and W. Bogaerts, in Proceedings of 9th IEEE International Conference on Group IV Photonics (IEEE 2012), pp. 234-236.

14. H. Yu, D. Ying, M. Pantouvaki, J. Van Campenhout, P. Absil, Y. Hao, Y. Yang, and X. Jiang, Opt. Express 22, 15178 (2014).

15. H. Yu, M. Pantouvaki, J. Van Campenhout, D. Korn, K. Komorowska, P. Dumon, Y. Li, P. Verheyen, P. Absil, L. Alloatti, D. Hillerkuss, J. Leuthold, R. Baets, and W. Bogaerts, Opt. Express 20, 12926 (2012).

16. M. Pantouvaki, P. Verheyen, G. Lepage, J. De Coster, H. Yu, P. De Heyn, P. Absil, and J. Van Campenhout, in Proceeding of 39th European Conference on Optical Communication (2013), paper We.2.B.2.

17. S. Xiao, M. H. Khan, H. Shen, and M. Qi, J. Lightwave Technol. 26, 228 (2008).

18. T. Kato and Y. Kokubun, J. Lightwave Technol. 24, 991 (2006). 